\title{
A study on the factors affecting the follow-up participation in birth cohorts
}

\author{
Bohyun Park ${ }^{1}$, Eun Jeung Choi ${ }^{1}$, Eunhee $\mathrm{Ha}^{1}$, Jong Hyuk $\mathrm{Choi}^{2}$, Yangho $\mathrm{Kim}^{3}$, Yun-Chul Hong ${ }^{4}$, \\ Mina $\mathrm{Ha}^{2}$, Hyesook Park ${ }^{1}$ \\ ${ }^{1}$ Department of Preventive Medicine, Ewha Womans University School of Medicine, Seoul; ${ }^{2}$ Department of Preventive Medicine, \\ Dankook University College of Medicine, Cheonan; ${ }^{3}$ Department Occupational and Environmental Medicine, Ulsan University \\ Hospital, Ulsan; ${ }^{4}$ Department of Preventive Medicine, Seoul National University College of Medicine, Seoul, Korea
}

Objectives A stable follow-up participation rate is a very important factor for validity in a cohort study. This study analyzed the factors that affect the participation rate at one hospital-based birth cohort in South Korean.

Methods The participants were recruited from the Mothers' and Children's Environmental Health study between 2006 and 2010. The analysis targeted 1751 mothers who participated in a birth cohort. We conducted analyses of general characteristics during pregnancy and those of infants at birth that affect the participation rate of the 6-month follow-up survey.

Results The participation rate for the 6-month follow-up survey was $60.4 \%$. The participation rate in the follow-up of the subsequent period decreased within a 5\% to $10 \%$ range compared to the number of subjects. The participation rate of premature infants was $16.9 \%$ lower than that of a full-term infant (52.6\% vs. 69.5\%). Analysis showed a $16.7 \%$ difference between the participation rate of low-birthweight infants in follow-ups $(53.7 \%)$ and the participation rate of infants with normal weight (70.4\%). The participation rate of mothers who were employed during pregnancy was significantly lower for the 6-month follow-up compared to the participation rate of mothers who were unemployed during pregnancy.

Conclusions In this study, factors such as premature birth, low-birthweight, and the employment status of the mother during pregnancy affected the participation rate of the follow-up survey for the birth cohort at six months. A specific strategy is needed to encourage survey participation for the high risk groups in the follow-ups.

Keywords: Birth cohort, Participation rate, Loss to follow-up

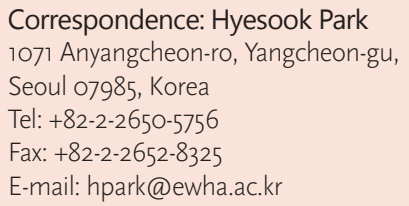

Received: April 21, 2016

Accepted: October 11, 2016

Published: November 22, 2016

This article is available from: http://e-eht.org/

\section{Introduction}

Exposure to environmental hazards during childhood increases future health risks. Diseases that occur due to such factors could lead to higher health care costs, which impact the societal disease burden. Because children have smaller body surface areas than adults while their volume of respiration and amount of food and drink intake are greater per weight, the effect of hazardous air and food on children is relatively great, making them the most vulnerable group in terms of exposure to hazardous substances containing chemical substances. In addition, because children are in a continuous level of physical, physiological, immunological, behavioral, and emotional growth, they are not only in an incomplete state of nervous, immune, and reproductive system development, but also in a vulnerable class that lacks knowledge of health risks when compared to adults [1].

Therefore, environmental health policies for children must be developed and implemented by considering the characteristics 
of children, which are different from those of adults. Considering the social impacts and increase in health care costs due to the increase in environmental diseases in children after industrialization, there is a great need for child-focused intervention studies and research on environmental health.

When the importance of studies for children is emphasized, birth cohort studies began in the early 2000s around the world. A birth cohort study is a longitudinal analysis in which subjects are followed and observed over an extended period of time, and the follow-up observation takes place from the prenatal, birth, infancy, and childhood, and if further extended, into adolescence and adulthood. The study carries out genetic and environmental research by collecting a variety of health data and gathering not only information on children, but also on parents, in order to assess the environmental risk factors.

A cohort study is a useful research method for determining the causality of the health effects of environmental exposure factors that influence disease outbreaks. In particular, the results of the birth cohort study could find health risk factors of early life and could be used as important evidence based data for establishing policies on early intervention for various disease outbreaks. Recently, birth cohorts have been established in many countries because of these advantages, and follow-up observation surveys have been carried out over long periods of time. Since there are limits for an individual researcher to conduct a birth cohort due to factors such as the long period of research time and the cost of follow-up observation, developed countries such as the US, Europe, and Japan plan and operate large-scale birth cohorts at the national level to carry out follow-ups over an extended period of time.

In order to get reliable study results from the birth cohorts, accuracy of the collected information during the follow-up observation survey must be maintained, and the quality management, such as encouraging continued engagement of the subjects in ongoing cohort, is also very important. The withdrawal of a study subject's cohort participation could act as a bias factor in study results. In particular, if there are selective instances of no response or follow-up failures depending on the occurrence of disease, such data could make it difficult to interpret the causal relationship between exposure and disease. Therefore, cohort research staff must consider the various factors that could affect the interpretation of the results starting from the cohort planning stage, and must make an effort to operate a stable cohort by minimizing the follow-up failure rates. In addition, in order to perform high quality cohort studies, it is necessary to investigate various factors that may influence the participation in follow-up, and continuous management of the derived factors will lead to reliable research results.

This study aimed to investigate the factors that affect the par- ticipation in follow-up surveys. We conducted analysis of the characteristics of infants at birth and the general characteristics of parents depending on the participation or non-participation of birth cohort subjects in the 6-month follow-up.

\section{Materials and Methods}

This study targeted 1751 mothers who agreed to participate in the Mothers' and Children's Environmental Health study. Follow-up observation surveys of the children birthed by the cohort subjects are being carried out continuously at 6, 12, 24, 36, $48,60,72,84$, and 96 months after birth. So far, follow-up observation surveys up to 36 months after birth have been completed. Follow-ups were carried out close to the month of survey in each period, after taking into account the birth dates of the subjects. Under unavoidable circumstances, follow-ups were carried out within the permitted range of one month before and after the month of survey in each period.

The following information was used in the analysis: 1) the general characteristics of parents gathered during pregnancy, 2) birth characteristics, and 3) after a follow-up, the participation or non-participation in surveys conducted at 6, 12, 24, and 36 months after birth. The participation rate in follow-ups for each period was assessed after defining follow-up participators as subjects whose anthropometry data was collected during follow-ups for each survey period.

We divided the subjects into two groups: one that participated in a 6-month follow-up and a group that did not for an analysis of general characteristics that affect the participation rate in followup observation surveys. In each group, the general characteristics during pregnancy and the birth characteristics of the children were analyzed. A descriptive analysis and $\chi^{2}$ test were conducted using the SAS version 9.3 (SAS Institute Inc,. Cary, NC, USA)

\section{Results}

Table 1 shows the analysis of the follow-up rate and the number of people in this cohort who participated in the follow-up surveys conducted until February 2015. The participation rate in the first 6-month follow-up was $60.4 \%$ out of 1751 total participants. The participation rate in subsequent follow-ups at 12 , 24 , and 36 months decreased within a $5 \%$ to $10 \%$ range compared to the participation rate at 6-month. When the participation rate of current follow-ups was compared to the participation rate in the previous period, there was a participation rate of more than $85.0 \%$. The participation rates in follow-ups at 12 , 24 , and 36 months by subjects who initially participated in the 6-month follow-up were 784 (74.1\%), 704 (66.5\%), and 612 
(57.8\%), respectively.

A gender ratio analysis of the 1516 participating children whose gender information was available indicated a similar rate of boys (792; 52.2\%) and girls (724; 47.8\%). There were no significant differences in gender regarding the participation in the 6-month follow-up (Table 2).

Comparison among subjects who participated in the 6-month follow-up after birth showed that there were significant differ-

Table 1. The number of follow-up subjects and the follow-up rate according to follow-up periods

\begin{tabular}{lccccc}
\hline Follow-up period & Mid-trimester & 6 mo & 12 mo & 24 mo & 36 mo \\
\hline No. of subjects & 1751 & 1058 & 927 & 825 & 726 \\
Participation rate (\%) & - & 60.4 & 52.9 & 47.1 & 41.5 \\
Participation rate (\%) & - & 60.4 & 87.6 & 89.0 & 88.0
\end{tabular}

aThe participation rate of the base survey participants.

${ }^{\mathrm{b} T}$ The participation rate of the previous follow-up participants.

Table 2. The related factors of the 6-month follow-up

\begin{tabular}{|c|c|c|c|}
\hline & $\begin{array}{l}\text { Participation in the } \\
\text { follow-up }(n=1058)\end{array}$ & $\begin{array}{l}\text { Non-participation in the } \\
\text { follow-up }(n=693)\end{array}$ & $p$-value \\
\hline \multicolumn{4}{|l|}{ Characteristics of the children } \\
\hline \multicolumn{4}{|l|}{ Gender } \\
\hline Men & $559(70.6)$ & $233(29.4)$ & \multirow[t]{2}{*}{0.52} \\
\hline Womene & $499(68.9)$ & $225(31.1)$ & \\
\hline \multicolumn{4}{|l|}{ Preterm delivery (wk) } \\
\hline Yes $(<37)$ & $41(52.6)$ & $37(47.4)$ & \multirow[t]{2}{*}{0.003} \\
\hline No $(\geq 37)$ & $988(69.5)$ & $433(30.5)$ & \\
\hline \multicolumn{4}{|l|}{ Low-birth weight (g) } \\
\hline Yes $(<2500)$ & $22(53.7)$ & $19(46.3)$ & \multirow[t]{2}{*}{0.03} \\
\hline No $(\geq 2500)$ & $1009(70.4)$ & $425(29.6)$ & \\
\hline \multicolumn{4}{|l|}{ Recruitment year } \\
\hline 2006-2007 & $510(54.1)$ & $432(45.9)$ & \multirow[t]{2}{*}{$<0.001$} \\
\hline $2008-2010$ & $548(67.8)$ & $261(32.3)$ & \\
\hline \multicolumn{4}{|l|}{ Characteristics of the mothers } \\
\hline Age of the mother during pregnancy (mean $\pm S D$ ) & $30.4 \pm 3.6$ & $30.1 \pm 3.9$ & 0.17 \\
\hline \multicolumn{4}{|l|}{ Marital status } \\
\hline Married/cohabitating & $1013(67.4)$ & $489(32.6)$ & \multirow[t]{2}{*}{0.03} \\
\hline Single/other & $16(48.5)$ & $17(51.5)$ & \\
\hline \multicolumn{4}{|l|}{ Employment status during pregnancy } \\
\hline Unemployed & $624(69.0)$ & $280(31.0)$ & \multirow[t]{2}{*}{0.01} \\
\hline Employed & $334(62.5)$ & $200(37.5)$ & \\
\hline \multicolumn{4}{|l|}{ Occupational group } \\
\hline Self-employed & $28(65.1)$ & $15(34.9)$ & \multirow[t]{5}{*}{0.19} \\
\hline Manager & $11(47.8)$ & $12(52.2)$ & \\
\hline Regular employee & $226(62.6)$ & $135(37.4)$ & \\
\hline Temporary or hourly employee & $34(72.3)$ & $13(27.7)$ & \\
\hline Other & $22(75.9)$ & $7(24.1)$ & \\
\hline \multicolumn{4}{|l|}{ Educational level } \\
\hline High school graduate & $323(67.7)$ & $154(32.3)$ & \multirow[t]{4}{*}{$0.16^{\mathrm{a}}$} \\
\hline Community college graduate & $155(73.1)$ & $57(26.9)$ & \\
\hline College graduate & $479(67.8)$ & $228(32.2)$ & \\
\hline Graduate school or above & $63(60.6)$ & $41(39.4)$ & \\
\hline \multicolumn{4}{|l|}{ Monthly income level (thousand Korean won) } \\
\hline Less than 1500 & $75(59.5)$ & $51(40.5)$ & \multirow[t]{6}{*}{0.03} \\
\hline 1500-1999 & $180(66.2)$ & $92(33.8)$ & \\
\hline 2000-2999 & $361(73.2)$ & $132(26.8)$ & \\
\hline 3000-3999 & $199(67.7)$ & 95 (32.3) & \\
\hline $4000-5999$ & $149(64.0)$ & $84(36.0)$ & \\
\hline More than 6000 & $40(66.7)$ & 20 (33.3) & \\
\hline
\end{tabular}

Values are presented as number (\%).

SD, standard deviation.

${ }^{a} p$ for trend. 
ences in the number of premature births and low-birthweight babies depending on whether participated or not. Analysis indicated that the participation rate in the 6-month follow-up was $69.5 \%$ for babies who were birthed after more than 37 weeks, but the participation rate was $52.6 \%$ (16.9\% point lower, $p=$ 0.003) for premature babies who were born earlier than 37 weeks. Similar results were observed for low-birthweight babies: the participation rate in the 6-month follow-up was $70.4 \%$ for babies who weighed more than $2500 \mathrm{~g}$ at birth while the participation rate was $53.7 \%$ ( $16.7 \%$ point lower, $p<0.05)$ for babies who weighed less than $2500 \mathrm{~g}$ at birth. Analysis of the participation rates according to recruitment years showed that the participation rate in surveys during the first two years (the first half of the recruitment) was $13.7 \%$ points higher than the participation rate during the last three years (the second half), which was a statistically significant result.

In order to compare the general characteristics of parents who participated in the 6-month follow-ups, the ages of the midpregnant (collected during pregnancy) and the socioeconomic statuses of the women were analyzed. In both groups, the average age at pregnancy was 30 years old, so there was not a significant difference. The marital status of the parents showed statistically significant relevance to the participation rate in the 6month follow-up. The participation rate was $67.4 \%$ for the mar$\mathrm{ried} /$ cohabitating group while the participation rate was $48.5 \%$ for the single/other group. The employment status of the mother during pregnancy also showed a significant relationship with the participation rate in the 6-month follow-up. The participation rate was $69.0 \%$ for mothers who were unemployed during pregnancy, while the participation rate was $62.5 \%$ (6.5\% point lower, $p=0.014$ ) for mothers who were employed during pregnancy. However, there was no statistical difference between the types of job held during pregnancy

In the case of household monthly income, the middle group (2 million Korean won to 3 million Korean won) showed the highest participation rate in the 6-month follow-up at $73.2 \%$, which indicated statistically significant relevance.

\section{Discussion}

Since the fetal origins hypothesis-which proposes that health hazard exposures during fetal life and infancy could influence the development of diseases in adulthood-has recently come to the fore, researchers are actively conducting life-course epidemiological studies. Life-course epidemiological studies are longterm studies that continuously observe the changes in health and observe the biological, behavioral, and psychosocial processes of the subjects from birth over the continuum of the sub- ject's lifetime. These studies mainly assess the influence of physical, environmental, and social exposure (which affect subjects over a lifetime, starting in utero through childhood, adolescence, and into adulthood) on the health and disease risks during adulthood. Such studies are mainly applied to chronic disease epidemiology.

A solidly built birth cohort allows life-course epidemiological studies to be conducted, which assess the causal effects of exposure to health risks during early life on the health conditions of adulthood. Even though a birth cohort has important role in the field of public health, there have been a lot of realistic difficulties in maintaining stable birth cohorts due to the time-consuming nature of a long-term study and the complications of continuously securing the needed resources for follow-up observations.

The Framingham Heart Study [2], which was established in the US - where cohort studies first started in the mid-20th century — had a $68 \%$ response rate, and the response rate was $24 \%$ for the 1989 Nurses' Health Study II [3]; however, there has been a trend of decreasing response rates in recent cohorts, indicating that recruiting cohort subjects and carrying out followups are becoming difficult in recent years. The response rate was $5.5 \%$ for a UK biobank cohort that was established in 2006 [4]. A similar tendency is observed regarding birth cohorts: for the 1958 birth cohort established in England [5], the first follow-up rate carried out in 1965 of 7-year-old children was $88.6 \%$, compared to the rate during pregnancy. Later, the follow-up rate for studies carried out until age 25 maintained a rate of over $95 \%$ compared to the rate of each previous period. In the case of a Denmark birth cohort that began in 1995 [6], the follow-up rate for the first follow-up at 6-month was 75.7\% compared to the recruitment rate of the early pregnancy period. Analysis showed that follow-up rates for later periods exceeded $90 \%$ in comparison to the rates of earlier periods. For the birth cohort in this study that began in 2006, the follow-up rate was $60.4 \%$ for the first follow-up at 6-month. The participation rates for subsequent follow-ups at 12,24 , and 36 months exceeded $85 \%$ compared to that of previous periods, showing a similar tendency when compared to overseas birth cohort studies. Even though it is difficult to increase the participation rate of birth cohort subjects in follow-ups and to keep a certain rate over time, maintaining a stable cohort is essential in order to attain reliable study results based on the birth cohort. For this purpose, researchers should use various approach methods even before establishing a cohort and prepare plans for step-by-step quality management. Moreover, there needs to be a policy to increase the participation rate in the first follow-up survey, knowing that there is relatively stable maintenance of the follow-up rates in birth cohort follow-ups after the first period. 
The characteristics of children and parents were compared depending on the participation or non-participation in the 6-month follow-up in order to examine the factors that influence participation in follow-up observation. Analysis shows that the non-participation rate was significantly high in groups with premature and low-birthweight babies, and the participation rate in the 6-month follow-up was low for babies born to mothers who were employed during pregnancy.

Children who are born prematurely and with low-birthweight are known to have higher incidence rates [7] of various diseases during their growth period and adulthood because they are more sensitive to environmental and social influences than are full-term babies. Regarding the cohort in this study, the low follow-up participation rate of premature and low-birthweight babies in the 6-month follow-up carried out in the form of a medical examination could be the result of a lack of motivation to participate in the check-up because of an increase in health care visits after birth by infants who are born with vulnerable characteristics. Therefore, it is necessary to prepare health management and intensive training programs targeting children of vulnerable health groups at the national level. Moreover, since low participation rates among premature and low-birthweight babies could affect the interpretation of the study results, measures must be prepared to manage follow-ups specialized for premature and low-birthweight groups, which would take the characteristics of each birth cohort.

Another factor that influenced the follow-up participation rate was the employment status of the mother during pregnancy. The participation rate in the 6-month follow-up was significantly lower for mothers who were employed during pregnancy. Working during pregnancy is an environmental factor that is known to have the possibility of causing negative effects on birth results (e.g., premature births and low-birthweight babies) because it triggers physical and mental stress [8]. Therefore, it could be thought of in relation to the decline in participation rate due to premature and low-birthweight babies. In addition, because mothers who were employed during pregnancy may continue employment after birth, time constraints could be the cause of low participation rates. Recently, the proportion of employment during and after pregnancy has been increasing in most countries, and the probability of working after pregnancy is higher for mothers who were employed during pregnancy. Employment after birth could act as a time and physical constraint against participation in follow-up observation surveys, and could later affect the interest and attitude in children's health. Moreover, considering the relationship between the decline in participation rates and marital status/monthly household income, there will need to be a plan that can increase the participation rate of vulnerable groups. In order to compare the characteristics of children and mothers depending on the participation rate in the 36-month follow-up, this study conducted an analysis with the same variables used for the analysis of the 6-month follow-up (data not shown). No statistically significant difference for any of the variables was found.

According to the study published by Lucas et al. [9], most European birth cohort studies are working to increase the participation rate of cohort subjects, and it has been reported that efforts have been made to increase participation through the operation of "research websites." The research teams of the cohorts sent newsletters, cards, and study results to subjects in order to maintain contact and some studies even utilized social networks, but there did not appear to be much active communication, such as taking the opinions of the subjects into account. Analysis in this study found that the following are barriers that affect the continuous participation in surveys: lack of trust in the quality of the study, ethical limitations, lack of resources, absence of priorities, negative experience in previous studies, etc. Moreover, a study on the effective maintenance of cohort research based on population [10] reported that financial incentive is the only strategy that maintains and increases participation. Therefore, it is very important to secure continuous funding that can maintain long-term cohort operations in order to maintain a stable birth cohort and enhance the follow-up rate. To achieve this, it is necessary to establish a support system at the national level.

In particular, providing various incentives is essential for vulnerable groups with low follow-up participation rates, since the rate of refusing participation is increasing due to a lack of specific incentives (e.g. receiving the results of check-ups that the subjects are interested in), which acts as a barrier to follow-up rates. Developing appropriate survey items and protocol for each lifecycle period could boost the appeal for cohort participation and increase understanding of priorities and importance, which will ultimately increase follow-up rates.

In addition, high priority must be assigned to follow-ups in order to increase the participation rate of subjects in the cohort. To achieve this, it is important to build a trusted relationship between researchers and subjects through active communication. Therefore, a strategy is needed in which both face-to-face communication and communication through mediums such as documents and the internet are used to actively accept queries and opinions from the subjects. Using the various methods mentioned above, preparation of plans to stimulate follow-up participation is expected to raise the quality of the birth cohort that is currently being conducted and yield high-quality populationbased study results. 


\section{Acknowledgements}

This study was carried out as the "The Mothers and Children's Environmental Health (MOCEH) study" project, which was funded by the Ministry of Environment in 2016.

\section{Conflict of Interest}

The authors have no conflicts of interest associated with material presented in this paper.

\section{ORCID}

Bohyun Park http://orcid.org/0000-0002-8538-9662

Eun Jeung Choi http://orcid.org/0000-0002-7698-3424

Eunhee Ha http://orcid.org/0000-0002-4224-3858

Jong Hyuk Choi http://orcid.org/0000-0002-8661-493X

Yangho Kim http://orcid.org/0000-0002-6462-0829

Yun-Chul Hong http://orcid.org/0000-0001-9010-7271

Mina Ha http://orcid.org/0000-0003-1011-9446

Hyesook Park http://orcid.org/0000-0002-9359-6522

\section{References}

1. Landrigan PJ, Carlson JE. Environmental policy and children's health. Future Child 1995;5(2):34-52.

2. Doll R, Hill AB. The mortality of doctors in relation to their smoking habits: a preliminary report. 1954. BMJ 2004;328(7455):15291533.

3. Nurses' Health Study. Nurses' Health Study: history [cited 2016 Nov 6]. Available from: http://www.nurseshealthstudy.org/aboutnhs/history.

4. Allena N, Sudlow C, Downey P, Peakman T, Danesh J, Elliott P, et al. UK biobank: current status and what it means for epidemiology. Health Policy Technol 2012;1(3):123-126.

5. Power C, Elliott J. Cohort profile: 1958 British birth cohort (National Child Development Study). Int J Epidemiol 2006;35(1):3441.

6. Andersen AM, Olsen J. The Danish National Birth Cohort: selected scientific contributions within perinatal epidemiology and future perspectives. Scand J Public Health 2011;39(7 Suppl):115-120.

7. Moraes $\mathrm{AB}$, Zanini RR, Riboldi J, Giugliani ER. Risk factors for low birth weight in Rio Grande do Sul State, Brazil: classical and multilevel analysis. Cad Saude Publica 2012;28(12):2293-2305.

8. Bonzini M, Coggon D, Palmer KT. Risk of prematurity, low birthweight and pre-eclampsia in relation to working hours and physical activities: a systematic review. Occup Environ Med 2007;64(4): 228-243.

9. Lucas PJ, Allnock D, Jessiman T. How are European birth-cohort studies engaging and consulting with young cohort members? BMC Med Res Methodol 2013;13:56.

10. Booker CL, Harding S, Benzeval M. A systematic review of the effect of retention methods in population-based cohort studies. BMC Public Health 2011;11:249. 